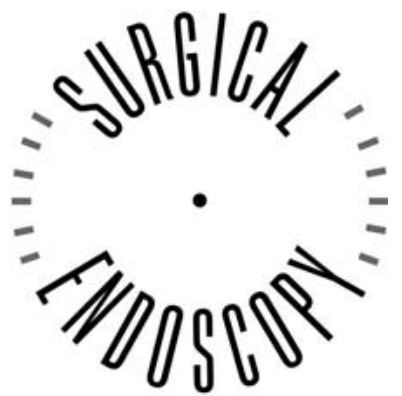

and Other Interventional Techniques

\title{
Video thoracoscopic surgery used to manage tuberculosis in thoracic surgery
}

\author{
M. Beshay, ${ }^{1}$ P. Dorn, ${ }^{1}$ J. R. Kuester, ${ }^{1}$ G. L. Carboni, ${ }^{1}$ M. Gugger ${ }^{2}$ R. A. Schmid ${ }^{1}$ \\ ${ }^{1}$ Division of General Thoracic Surgery, University Hospital Berne, Berne, 3010, Switzerland \\ ${ }^{2}$ Institute of Pathology, University Hospital Berne, Berne, 3010, Switzerland \\ Received: 7 June 2004/Accepted: 11 February 2005/Online publication: 23 June 2005
}

\begin{abstract}
Background: The aim of this study was to evaluate the indications and results of video-assisted thoracic surgery (VATS) for the management of tuberculosis in 10 patients with unusual clinical and radiologic presentation for the disease.

Methods: From March 2000 to March 2002, 96 diagnostic VATS operations for unclear thoracic lesions were performed at the authors' institution. Their final diagnosis for $10(10.4 \%)$ of these patients was tuberculosis. The suspected preoperative diagnoses were pancoast tumour $(n=1)$, pericardial effusion $(n=1)$, pleural mesothelioma $(n=1)$, pleural empyema $(n=2)$, mediastinal lymphoma $(n=1)$, and lung cancer $(n=4)$.

Results: For all the patients, the diagnosis of tuberculosis was achieved by VATS. The duration of drainage was 2.5 days. There have been neither morbidity nor mortality since surgery. The hospital stay was 3 to 5 days.

Conclusion: Thoracoscopy is a safe and effective procedure for the management of tuberculosis. Tuberculosis should be kept in mind during the differential diagnosis of unknown thoracic lesions, and also for patients who live in economically well developed countries and are not immune compromised.
\end{abstract}

Key words: Tuberculosis — Video-assisted thoracic surgery - Lung

Tuberculosis (TB) is still one of the leading causes of death in developing countries. Also, incidence of tuberculosis has been increasing since 1985 in the economically well-developed countries. This could be attributable to a higher number of immune-compro-

Presented at the 10th annual meeting of the European Society of Thoracic Surgeons, Istanbul, Turkey, 26-28 October 2002

Correspondence to: $\mathrm{R}$. A. Schmid mised patients, especially human immuno deficiency virus (HIV) seropositive patients, or to increasing immigration $[2,10,11,13]$. Thus, it appears that the clinical and radiologic presentation of tuberculosis has changed to more unusual manifestations. With the dramatic improvement of video-assisted thoracic surgery (VATS), this approach for the diagnosis of different thoracic lesions is recommended as standard, and is becoming popular for the diagnosis of TB [15].

Hans Christian Jacobaeus [4], a Swedish internist, is generally known as the first to undertake an endoscopic exploration of the thorax in 1910 to break pleural adhesions with the patient under local anesthesia to facilitate collapse therapy for pulmonary tuberculosis. In French, at least, the term "thoracoscopie" (exploration de la cavité thoracique) was well known long before Jacobaeus [6]. In 1865, Fancis Richard Cruise, an Irish physician, performed an endoscopic examination of a chest fistula using a binocular device [1], but Jacobaeus was the first to use the term "thoracoscopy" [3, 5].

After the introduction of streptomycin in 1945 and other effective antibiotic therapies for tubercle bacilli, the tendency to use thoracoscopy for patients with TB was declining. Pleural biopsy and empyema irrigation were the main remaining roles for thoracoscopy in the management of tuberculosis.

Retrospectively, we analyzed the incidence of TB among a group of patients for whom the diagnosis of TB failed to be established using the usual methods of investigations. The use of VATS as a safe and effective method was helpful for both the diagnosis and the management of some TB complications.

\section{Patients and methods}

From March 2000 to March 2002, 96 VATS operations for unknown thoracic lesions were performed at our institution. Of these patients, 96 had either primary bronchial carcinoma or metastases in the lung, 12 had sarcoidosis, 11 had chronic localized inflammatory lesions, 7 had hamartoma, and 10 had a final diagnosis of TB. Histologic and mi- 


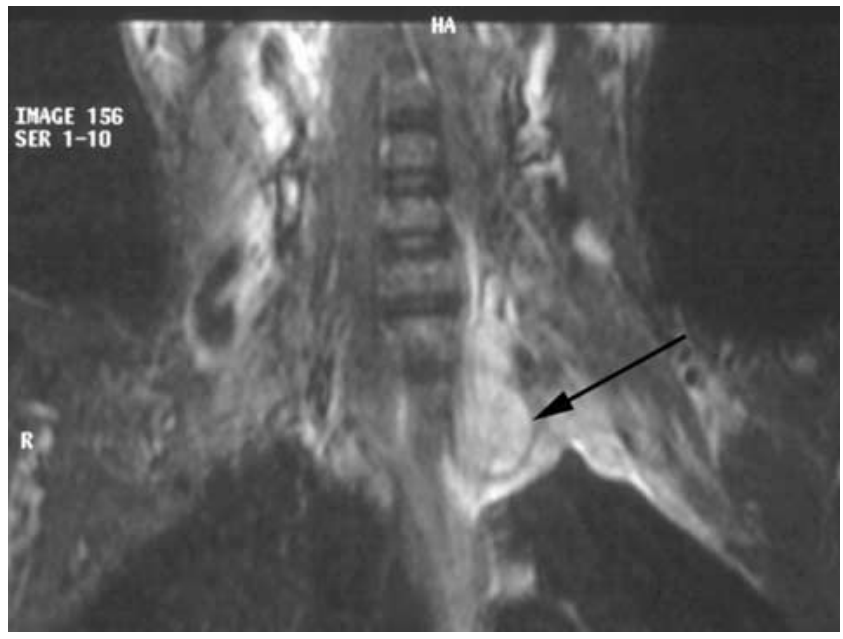

Fig. 1. A 48-years old man presented with features of Pancoast tumor. His magnetic resonance imaging showed the lesion with infiltration of the pleura (arrow).



Fig. 2. A 27-year-old man with fever and signs of upper venous flow obstruction whose computed tomography scan of the chest shows a large pericardial effusion (arrow).

crobiologic studies were done from all the patients. Microbiologic culture and polymerase chain reaction examination were performed only nonmalignant tissues, especially those with granulomatous lesion.

We retrospectively studied the patients for whom the final diagnosis was tuberculosis. Records were reviewed for age, sex, ethnic origin, history, clinical symptoms, site of the lesions, HIV serologic results, surgical procedure, and postoperative events. Sputum culture performed for 7 of 10 patients showed no Mycobacterium tuberculosis. Bronchoscopic examination with bronchoalveolar lavage was performed for six patients, showing negative results for all of them. Tuberculin skin testing (Mantoux test) performed for five patients showed positive results for two. The preoperative suspected diagnoses were. Pancoast tumor $(n=1)$ (Fig. 1), pericardial effusion $(n=1)$ (Fig. 2), pleural mesothelioma $(n=1)$, pleural empyema $(n=2)$ (Fig. 3$)$, mediastinal lymphoma $(n=1)$, and lung cancer $(n=4)$ (Fig. 4$)$.

General anesthesia with selective one-lung ventilation was used. Single-dose second-generation cephalosporin was given for prophylaxis in all cases. All the patients had video-assisted thoracoscopy through the standard three-ports procedures using one rigid trocar for the 7-mm video camera, and the other two flexible trocars for the use of the instruments. The patient was lying on the healthy side in the lateral position. For wedge resection of the lung in four cases, the ventral incision was elongated to admit the 20 - $\mathrm{mm}$ rubber trocar for the parenchymal stapler (Endo GIA 45; Ethicon Endosurgery; Johnson \& Johnson, 4545 Creek Road, Cincinnati, OH, USA).

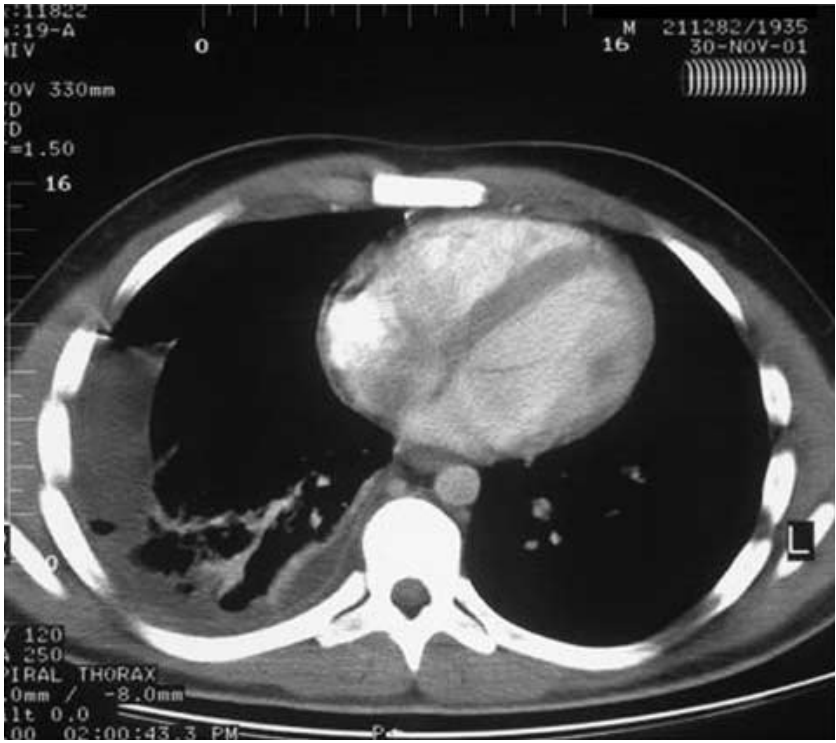

Fig. 3. A 20 -year-old athletic patient presented with cough and fever. Computed tomography scan of the chest shows right pleural empyema with trapped lung.
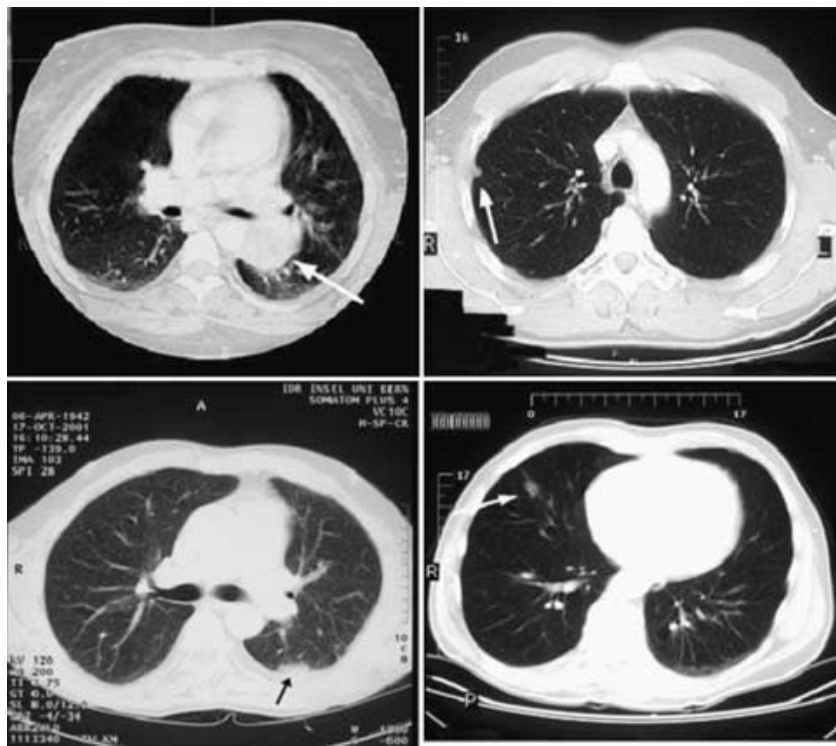

Fig. 4. Computed tomography scans of four different patients with different masses in the lung.

Through the same incision, the specimens were removed. Lateral thoracotomy was performed in the sixth intercostal space for two patients. At the end of the procedure, two chest tubes were placed at the most anterior insertion sites. All the patients were extubated immediately after surgery. All the chest tubes were withdrawn on postoperative days 2 and 3. Postoperative analgesia was ensured with a patient-controlled analgesia system in four patients and with a peridural analgesia system in six patients. Information was obtained from our outpatient clinic, the patient's family doctor, and by telephone interview.

\section{Results}

The median age of the patients was 51.7 years (range, 19-79 years). Eight of the patients were men, and two 
Table 1. Applications of video-assisted thoracic surgery for managing thoracic presentations of tuberculosis (TB)

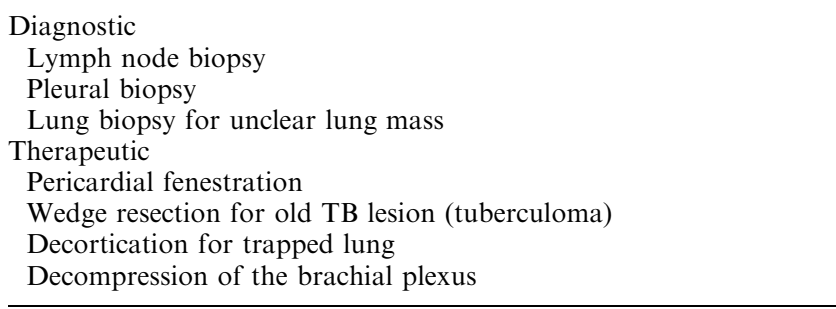

were women. Ethnically, the patients included seven Swiss patients $(70 \%)$, two Africans (who had lived in Switzerland, respectively, 3 and 4 years), and one Asian. One patient had hepatitis, showing A-,B-, and C-positive serology. No patient had a positive serology for HIV. The HIV test was performed for three patients with a suspect history, and the other seven patients did not have any risk factors. Eight of the patients had had an unremarkable previous medical history, except for one patient who had a history of chronic obstructive pulmonary disease and one patient who had been receiving steroids $(20 \mathrm{mg} /$ day $)$ because of his known polyarthritis with polymyositis for the preceding 6 years. Two patients $(20 \%)$ presented with pain in the shoulder region and in the thoracic wall, and two patients had fever with cough and expectoration. Another two patients had unspecific symptoms. Four patients $(40 \%)$ had no symptom, and the lesion had been discovered after a routine chest x-ray examination.

There was neither morbidity nor mortality during or after surgery. The diagnosis was achieved by VATS, expect for one patient with pericardial effusion in whom minithoracotomy was performed because of strong adhesions between the lung and the pericardium. Two patients needed conversion to lateral thoracotomy to ensure complete decortication of the lung after biopsies for histologic and microbiologic examinations. In our series, the VATS procedure was performed for diagnostic und therapeutic strategies (Table 1). The operative time for VATS ranged between 60 and $90 \mathrm{~min}$ (mean, $75 \mathrm{~min}$ ).

The diagnosis of tuberculosis was confirmed by histopathologic demonstration of caseous necrosis with chronic inflammatory and granulation tissue reaction and or bacteriologic examination (smear or cultures) demonstrating $M$. tuberculosis. For three patients, TB was confirmed by polymerase chain reaction, and for five patients, $M$. tuberculosis was found at microbiological examination. For nine patients, triple tuberculostatic therapy was started for 6 to 9 months. One patient did not receive antituberculous therapy because of the histologic findings, which showed old tuberculoma. The lesions were removed completely with wedge resection. Eight patients were discharged from the hospital $24 \mathrm{~h}$ after removal of the last chest tubes. The duration of the chest drainage was between 2 and 4 days (mean, 2.5 days). The hospital stays were 3 to 5 days (mean, 4.7 days). Two patients (20\%) had to stay longer (15 and 17 days, respectively) for social reasons.

\section{Discussion}

Tuberculosis remains a serious worldwide health problem. The incidence of tuberculosis has been increasing again in Western countries. Moreover, there often are difficulties in determining the diagnosis of $\mathrm{TB}$ on radiologic examinations because of unusual clinical and radiographic findings. Some of these atypical clinical lesions, such as lack of cavitations, mediastinal adenopathy, and equal frequency of distribution between the upper and lower lobes, were reported 10 years ago, especially in HIV seropositve patients at an advanced stage [18].

In our series of patients, we found some atypical presentations such as large pericardial effusion and paravertebral mass mimicking a superior sulcus tumor without pulmonary manifestations. For these cases, magnetic resonance imaging was helpful because it showed the changes in the surrounding soft tissues and compression of the nearby nerves (e.g., brachial plexus) [14]. Diagnostic approaches to the indeterminate solitary pulmonary nodule have been bronchoscopy, transthoracic needle aspiration biopsy, and thoracoscopy. Indeed, bronchoscopy is helpful for large central and intraluminal lesions, but has a low diagnostic yield, approximately $10 \%$ for small, peripheral nodules. Transthoracic needle aspiration biopsy has a reported diagnostic accuracy ranging from $43 \%$ to $97 \%$ for malignant lesions, but it is of limited value for obtaining a definitive diagnosis in the case of benign lesions [7, 9]. In cases of peripheral solitary pulmonary nodule or extrapulmonary lesions, thoracoscopy is the most helpful method for establishing the diagnosis, especially if the lesion is not reachable with transthoracic needle aspiratioin biopsy (e.g., in patients with pericardial or pleural pathologies). In fact, the diagnostic accuracy of thoracoscopic biopsy is as good as that of open biopsy and is playing a major role in the management of thoracic lesions. As a minimal invasive procedure, VATS is well accepted by the patients because it has a low surgical burden and minimal morbidity with easier pain control. Therefore, the hospital stay is significantly shorter after VATS. Thoracotomy, on the other hand, has significant morbidity with a higher surgical burden.

In general, both the operative time and hospital stay are longer after thoracotomy than after VATS. The efficient diagnosis of tuberculosis requires a high index of suspicion so that specimens can be obtained for bacteriologic and histologic examinations. Although six patients had symptoms not directly related to $\mathrm{TB}$, and all the preoperative investigations for $\mathrm{TB}$, including the Mantoux test (with positive results only for two patients), sputum examination bronchoalveolar and lavage yielded negative results, TB was confirmed histologically and microbiologically in 10 patients. Thus, TB often is difficult to diagnose with the ordin- 
ary methods, especially if the lesion is peripheral or outside the lung [8]. Therefore, in such cases, VATS plays an important role in confirming or ruling out TB depending on the final histologic and microbiologic examinations.

Although surgery is of limited value for the treatment of active tuberculosis, it still plays a complementary role for selected patients $[12,16]$. In the current study, surgery was indicated in three situations as a method of treatment: drainage of a large pericardial effusion, decortication of a trapped lung, and Decompression of brachial plexus. Wedge resection provides a definitive diagnosis essential for the further management of old tuberculoma lesions using VATS. Wedge resection usually is curative, although the patient should take antituberculous drugs for 2 to 4 months postoperatively $[9,12,16]$. Among all the patients there was no perioperative mortality or morbidity and a relatively short hospitalization time.

We conclude that thoracoscopy is a safe and effective approach for the diagnosis and treatment of $\mathrm{TB}$ or its complications. Video-assisted thoracoscopy is a less invasive and cost more-effective procedure than thoracotomy and more acceptable by patients. Therefore, VATS may help in the early diagnosis and treatment of tuberculosis, which is important for minimizing progression of the disease and the development of complications [17]. Tuberculosis should be always kept in mind as a differential diagnosis for unknown thoracic lesions and for immune-competent patients who are not immigrants from endemic areas.

\section{References}

1. Cruise FR (1865) The endoscope as an aid to the diagnosis and treatment of disease. Br Med J 8: 345-347
2. Frieden TR, Sterling T, Pablos-Mendez A, Kilburn JO, Cauthen GM, Dooley SW (1993) The emergence of drug resistance in New York City. N Engl J Med 328: 521-526

3. Jacobaeus HC (1911) Kurze Uebersicht über meine Erfahrungen mit der Laparo-Thorakoskopie. Münchn Med Wochenschr 58: 2017-2019

4. Jacobaeus HC (1910) Ueber die Möglichkeit die Zystoskopie bei untersuchung seröser höhlungen anzuwenden. München Med Wochenschr 57: 2090-2092

5. Jacobaeus HC (1912) Ueber Laparo-und Thorakoskopie. Beitr Klein Tuberk 25: 185-186

6. Larousse P (1876) Grand dictionnaire universel, vol. 15 Administration du Grand Dictionnaire Universel, Paris, p 154

7. Lau SK, Wie WI, Hsu C, Engzell UCG (1990) Efficacy of fineneedle aspiration cytology in the diagnosis of tuberculous cervical lymphadenopathy. J Laryngol Otol 104: 24-27

8. Long R, Maycher B, Scalcini M, Manfreda J (1991) The chest roentgenogram in pulmonary tuberculosis patients seropositive for HIV type 1. Chest 99: 123-127

9. Mack MJ, Hazelrigg SR, Landreneau RJ, Acuff TE (1993) Thoracoscopy for the diagnosis of the indeterminate solitary pulmonary nodule. Ann Thorac Surg 56: 825-832

10. Meduri UG, Stein DS (1992) Pulmonary manifestations of acquired immunodeficiency syndrome. Clin Infect Dis 14: 98-113

11. Narian JP, Raviglone MC, Kochi A (1992) HIV-associated tuberculosis in developing countries: epidemiology and strategies for prevention WHO, Geneva, Switzerland: World Health Organization, WHO/TB92.164, p 164

12. Reed CE, Parker EF, Crawford FA (1989) Surgical resection for complications of pulmonary tuberculosis. Ann Thorac Surg 48: 165-167

13. Selwyn PA, Sckell BM, Alcabes P, Friedland GH, Klein RS, Schoenbaum EE (1992) High risk of active tuberculosis in HIVinfected drug users with cutaneous energy. JAMA 268: 504-509

14. Sharif HS, Morgan JL, Al Shahied MS (1995) The role of CT and MR imaging in the management of tuberculous spondylitis. Radiol Clin North Am 33: 787-804

15. Treasure RL, Seaworth BJ (1995) Current role of surgery in mycobacterium tuberculosis. Ann Thorac Surg 59: 1405-1408

16. Whyte RI, Geegan SP, Kaplan DK, Evans CC, Donnelly RJ (1989) Recent surgical experience for pulmonary tuberculosis. Respir Med 83: 357-362

17. Yim APC (1996) The role of video-assisted thoracoscopic surgery in the managemnt of pulmonary tuberculosis. Chest 110: 829-832

18. Yim APC, Mohammad BI, Tak WL (1999) Thoracoscopic surgery for pulmonary tuberculosis. World J Surg 23: 1114-1117 